\title{
An efficient method for the synthesis of 2,4,5-trisubstituted imidazoles using lactic acid as promoter
}

\author{
Jayant Sonar ${ }^{1} \cdot$ Sandeep Pardeshi ${ }^{1} \cdot$ Shrikant Dokhe ${ }^{1} \cdot$ Rajendra Pawar $^{4} \cdot$ Kiran Kharat $^{5} \cdot$ Ashok Zine $^{2}$.

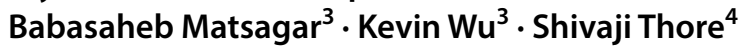

(c) Springer Nature Switzerland AG 2019

\section{Abstract}

Synthesis of 2,4,5-trisubstituted imidazole compounds from an aromatic aldehyde, benzil and ammonium acetate is demonstrated using biodegradable lactic acid at $160^{\circ} \mathrm{C}$. This method is a simple, environmentally benign, and works for aromatic aldehyde containing electron donating and electron withdrawing groups.

\section{Graphic abstract}

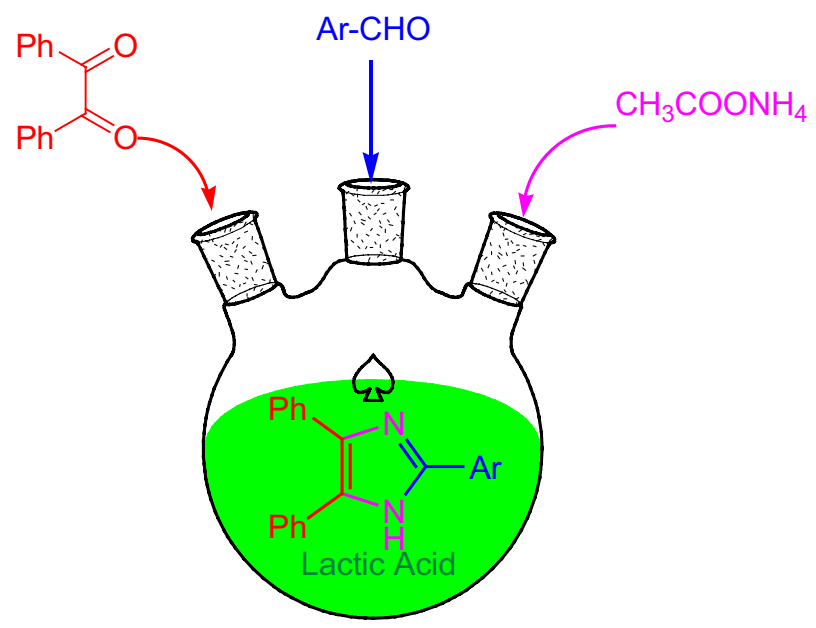

Keywords Lactic acid · Promotor · Green solvent · 2,4,5-Trisubstituted imidazoles

$\triangle$ Shivaji Thore, shivajiraothore@rediffmail.com | 'Department of Chemistry, Vinayakrao Patil Mahavidyalaya, Vaijapur, Aurangabad, Maharashtra 423 701, India. ${ }^{2}$ Department of Chemistry, Sunderrao Solunke Mahavidyalaya, Majalgaon, Dist. Beed, Maharashtra 431 131, India. ${ }^{3}$ Chemical Engineering Department, National Taiwan University, Taipei 106, Taiwan. ${ }^{4}$ Department of Chemistry, Deogiri College, Station Road, Aurangabad, Maharashtra 431 005, India. ${ }^{5}$ Department of Biotechnology, Deogiri College, Station Road, Aurangabad, Maharashtra 431 005, India. 


\section{Introduction}

Imidazole is an important core organic molecule. It is found in many naturally occurring compounds like vitamin $\mathrm{B}_{12}$, histidine [1], histamine, pilocarpine alkaloids, nucleic acid bases, and biotin [2-5] Imidazole is also found to be an active part of antifungal compounds like clotrimazole, ketoconazole, miconazole, isoconazole, econazole [6]. Along with this, it is also showing good activity as herbicide [7], plant growth regulator [8], anti-epileptic [9], anticonvulsant [10], anti-inflammatory, analgesic [11], anticancer, etc. [12, 13]. Also, imidazoles are found as the main core molecule in drugs like Omeprazole, Pimobendan, Losarton, Olmesartan, Eprosartan, and Trifenagrel [14].

In organic synthesis, the product yield and reaction time are extremely important. The increase in reaction steps results in a decrease in final product yield and increase in total reaction time. Multicomponent reactions help to solve this problem. By novel developing multicomponent reaction strategies, synthesis of the desired product in the one-pot method is possible thereby increases the product yield and reducing reaction time required for the reaction. Thus, it can be considered as a greener way in organic synthesis and have attracted significant attention from many researchers in recent years [15].

Various methods are reported in the literature for the one-pot synthesis of imidazole derivatives from benzil, aromatic aldehyde, and ammonium acetate. Heterogeneous catalyst such as Lewis acids $\left(\mathrm{NiCl}_{2} \cdot 6 \mathrm{H}_{2} \mathrm{O}\right.$ [16], $\mathrm{Ce}\left(\mathrm{NH}_{4}\right)_{2}\left(\mathrm{NO}_{3}\right)_{6}$ [17], $\mathrm{ZrCl}_{4}$ [18], $\mathrm{CrCl}_{3} \cdot 6 \mathrm{H}_{2} \mathrm{O}$ [19], $\mathrm{Pb}(\mathrm{OAc})_{2}$ [20], etc.), nano particles $\left(\mathrm{MgAl}_{2} \mathrm{O}_{4}\right.$ [21], nanorod vanadate sulfuric acid [22], magnetic $\mathrm{Fe}_{3} \mathrm{O}_{4}$ [23], etc.) are reported for imidazole synthesis. $\mathrm{CrCl}_{3} \cdot 6 \mathrm{H}_{2} \mathrm{O}$ and $\mathrm{Pb}(\mathrm{OAc})_{2}$ are toxic in nature. lonic liquids (ILs) [24-28] are also reported for the efficient synthesis of substituted imidazoles. However, ILs are expensive and recyclability of ILs are difficult [29]. Organic solvents like glycerol [30], Pri2NEt [31], PEG [32], acetic acid [33, 34], Glyoxylic acid [35], natural acids [36] are reported for the synthesis of imidazoles.

The available reported method for the synthesis of substituted imidazoles suffers from drawbacks such as the catalysts used for synthesis are either toxic or expensive and requires harsh reaction condition. To overcome these problems, we employed inexpensive, biodegradable, environmentally friendly green solvent lactic acid $[37,38]$ for the synthesis of 2,4,5-triaryl-1H-imidazoles. The synthesis method does not require any sophisticated assembly, and lactic acid used in this reaction acts as promoter and also solvent. Lactic acid is obtained from fermentation of carbohydrates, it is easy to handle and readily available. Lactic acid is reported as organocatalyst in several organic reactions [39-48]. In the present method we are reporting

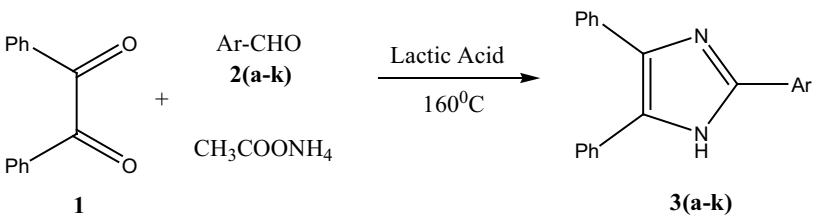

Fig. 1 General reaction scheme for the synthesis of 2,4,5-trisubstituted imidazole

Table 1 Effect of solvent for the synthesis of 2,4,5-triphenyl-1H-imidazole

\begin{tabular}{llllll}
\hline $\begin{array}{l}\text { Entry } \\
\begin{array}{l}\text { Lactic } \\
\text { acid } \\
(\mathrm{mL})\end{array}\end{array}$ & $\begin{array}{l}\text { Solvent } \\
(10 \mathrm{~mL})\end{array}$ & Time $(\mathrm{min})$ & Temp. $\left({ }^{\circ} \mathrm{C}\right)$ & Yield $(\%)$ \\
\hline 1 & 0.1 & Water & 180 & 50 & 37 \\
2 & 0.1 & Acetonitrile & 180 & 50 & 30 \\
3 & 0.1 & Methanol & 180 & 50 & 42 \\
4 & 0.1 & DMF & 180 & 50 & 45 \\
5 & 0.1 & DCM & 180 & 50 & 20 \\
6 & 0.1 & Dioxane & 180 & 50 & 32 \\
7 & 0.1 & Ethanol & 180 & 50 & 55 \\
8 & 0.1 & Ethanol & 180 & 70 & 72 \\
9 & 0.2 & Ethanol & 240 & Reflux & 72 \\
10 & 0.4 & Ethanol & 240 & Reflux & 75 \\
\hline
\end{tabular}

Benzaldehyde $1 \mathrm{mmol}$, ammonium acetate $3 \mathrm{mmol}$, benzil $1 \mathrm{mmol}$

lactic acid for the synthesis of 2,4,5-trisubstituted imidazole compounds from an aromatic aldehyde, benzil, and ammonium acetate under milder reaction conditions $\left(160^{\circ} \mathrm{C}\right)$ (Fig. 1).

\section{Result and discussion}

Initially, benzaldehyde was selected as a prototype for the reaction with benzil and ammonium acetate to study the catalytic activity of lactic acid. The reaction conditions were optimized to efficiently synthesize the 2,4,5-triphenyl-1H-imidazole in solvent-free condition with the catalytic amount of lactic acid, but the results were not satisfactory even at higher temperatures. Hence, we added lactic acid in various solvents and reactions were performed with different temperature (Table 1). Through the experimentation, we achieved $55 \%$ of product yield in ethanol-lactic acid system. The better results are witnessed at $70^{\circ} \mathrm{C}(3 \mathrm{~h}$ ) with 1:1:3 equivalent proportions of benzil, an aromatic aldehyde, and ammonium acetate, respectively.

The result shows that with an increase in the quantity of lactic acid in ethanol is not exhibiting improvement in the product yield (Table 1). Further, we conducted reactions 
Table 2 Optimization of reaction parameters for the synthesis of 2,4,5-triphenyl-1 $\mathrm{H}$-imidazole using lactic acid

\begin{tabular}{lllll}
\hline Entry & $\begin{array}{l}\text { Lactic acid } \\
(\mathrm{mL})\end{array}$ & $\begin{array}{l}\mathrm{NH}_{4} \mathrm{OAc} \\
(\mathrm{mmol})\end{array}$ & Temp. $\left({ }^{\circ} \mathrm{C}\right)$ & Yield $(\%)$ \\
\hline 1 & 0.5 & 2 & 100 & 62 \\
2 & 1.0 & 2 & 130 & 70 \\
3 & 1.0 & 2.5 & 130 & 74 \\
4 & 1.0 & 3.0 & 130 & 75 \\
5 & 1.0 & 2.5 & 160 & 90 \\
6 & 1.0 & 3.0 & 160 & 89 \\
7 & 1.0 & 2.5 & 170 & 92 \\
\hline
\end{tabular}

Benzaldehyde $1 \mathrm{mmol}$, benzil $1 \mathrm{mmol}, 180 \mathrm{~min}$

Table 3 Comparison of natural acids for the synthesis of 2,4,5-trisubstituted imidazoles

\begin{tabular}{lllll}
\hline Entry & Acid & Temp. $\left({ }^{\circ} \mathrm{C}\right)$ & Time $(\mathrm{min})$ & Yield $(\%)^{\mathrm{a}}$ \\
\hline 1 & Citric & 160 & 20 & $76[36]$ \\
2 & Fumaric & 290 & 20 & $65[36]$ \\
3 & Malic & 140 & 20 & $78[36]$ \\
4 & Malonic & 140 & 20 & $80[36]$ \\
5 & Oxalic & 110 & 20 & $77[36]$ \\
6 & Succinic & 190 & 20 & $68[36]$ \\
7 & Tartaric & 180 & 20 & $75[36]$ \\
8 & Lactic & 160 & 180 & 92 \\
\hline
\end{tabular}

a 4-Cl- Benzaldehyde

only in the lactic acid, and the result shows an increment in the product yield in $1 \mathrm{~mL}$ lactic acid.

The proportions of lactic acid and ammonium acetate were varied at different temperature (Table 2 ). This showed the best product yield with $1 \mathrm{~mL}$ of lactic acid, $2.5 \mathrm{mmol}$ of ammonium acetate at $160{ }^{\circ} \mathrm{C}$ (Table 2 , entry 5). The obtained results were compared with other natural acids (Table 3) [36] for the synthesis of imidazoles and it shows that our method is efficient for better product yields using lactic acid.

As shown in Table 4, this catalytic system works well for both aromatic aldehydes containing electron donating and electron withdrawing groups. For 4-nitro benzaldehyde (entry f) $83 \%$ yield was obtained in $180 \mathrm{~min}$ while for 4-(dimethylamino)benzaldehyde $91 \%$ yield was obtained in 150 min (entry h). It means there is no specific effect of electron density on the product yield. However, the desired reaction time can be changed depending on the type of aldehyde used for the reaction. The probable mechanism of reaction is depicted in Fig. 2 .

\section{Experimental}

All the melting points were recorded by open capillary method and are uncorrected. IR spectra were recorded on Shimadzu IR Affinity 1 spectrophotometer in $\mathrm{KBr}$ disc. ${ }^{1} \mathrm{H}$ NMR were recorded on a BRUKER AVANCE II $400 \mathrm{MHz}$ spectrometer in DMSO $d^{6}$, chemical shifts are in ppm relative to TMS. Mass spectra were taken on a Macro mass spectrometer by electron spray method (Es). The structures of various synthesized compounds were assigned on the basis of spectral studies and it has been reported in experimental protocols. The progress of reaction was monitored on Alumina coated TLC plates in ethyl acetate and $\mathrm{n}$ - hexane system.

General experimental procedure: In $10 \mathrm{~mL}$ round bottom flask aromatic aldehyde $(1 \mathrm{mmol})$, benzil $(1 \mathrm{mmol})$ and ammonium acetate $(2.5 \mathrm{mmol})$ were added. To this reaction mixture, $1 \mathrm{~mL}$ of lactic acid was added and then reaction mixture was heated at $160^{\circ} \mathrm{C}$ for an appropriate time (Table 4). The progress of reaction was monitored with the help of TLC (hexane: ethyl acetate 8:2 v/v). After completion of reaction, the mixture was poured over crushed ice ( $100 \mathrm{~g}$ and neutralized by saturated solution of sodium carbonate. The solid obtained was filtered and purified by column chromatography using hexane-ethyl acetate as eluent $(9: 1 \mathrm{v} / \mathrm{v})$.

Characterization:

1-(4-(4,5-diphenyl-1H-imidazol-2-yl)phenyl)piperidine (3j):

IR $\left(\mathrm{KBr}, \mathrm{cm}^{-1}\right): 3080,2880,1600-1500$

${ }^{1} \mathrm{H}$ NMR $\left(400 \mathrm{MHz}, \mathrm{DMSO}-\mathrm{d}_{6}, \delta, \mathrm{ppm}\right): 1.56-1.62(\mathrm{~m}, 6 \mathrm{H}$, $\left.3 \mathrm{CH}_{2}\right), 3.21-3.23\left(\mathrm{t}, 4 \mathrm{H}, 2 \mathrm{CH}_{2}\right), 6.99(\mathrm{~d}, \mathrm{~J}=8 \mathrm{~Hz}, 2 \mathrm{H}, \mathrm{Ar}-\mathrm{H})$, 7.21-7.55 (m, 10H, Ar-H), $7.94(\mathrm{~d}, \mathrm{~J}=8 \mathrm{~Hz}, 2 \mathrm{H}, \mathrm{Ar}-\mathrm{H}), 12.29$ $(\mathrm{s}, 1 \mathrm{H}, \mathrm{NH})$

${ }^{13} \mathrm{C}$ NMR $\left(100 \mathrm{MHz}\right.$, DMSO- $\left.\mathrm{d}_{6}\right): 23.90,25.10,48.87$, $114.98,120.24,126.25,127.05,127.38,128.08,128.24$, $128.52,129.49,129.55,131.31,135.52,136.59,146.19$, 151.23

ESI-MS (m/z): $379.49(\mathrm{M}+1)$

4,5-diphenyl-2-(4-(pyrrolidin-1-yl)phenyl)-1H-imidazole (3k):

IR $\left(\mathrm{KBr}, \mathrm{cm}^{-1}\right): 3100,2930,1600-1500$

${ }^{1} \mathrm{H}$ NMR $\left(400 \mathrm{MHz}\right.$, DMSO-d $\left.{ }_{6}, \delta, \mathrm{ppm}\right): 1.96\left(\mathrm{t}, 4 \mathrm{H}, 2 \mathrm{CH}_{2}\right)$, $3.28\left(\mathrm{t}, 4 \mathrm{H}, 2 \mathrm{CH}_{2}\right), 6.61(\mathrm{~d}, \mathrm{~J}=8 \mathrm{~Hz}, 2 \mathrm{H}, \mathrm{Ar}-\mathrm{H}), 7.20-7.56(\mathrm{~m}$, $10 \mathrm{H}, \mathrm{Ar}-\mathrm{H}), 7.91(\mathrm{~d}, \mathrm{~J}=8 \mathrm{~Hz}, 2 \mathrm{H}, \mathrm{Ar}-\mathrm{H}), 12.29(\mathrm{~s}, 1 \mathrm{H}, \mathrm{NH})$

${ }^{13} \mathrm{C}$ NMR(100 MHz, DMSO- $\left.d_{6}\right): 24.95,47.21,11.37$, $117.52,126.20,126.41,126.89,127.03,127.30,128.06$, $128.21,128.52,131.46,135.60,136.45,146.71,147.59$

ESI-MS (m/z): $365.4(\mathrm{M}+1)$ 
Table 4 Synthesis of 2,4,5-trisubstituted imidazoles 3(a-k) using lactic acid

\begin{tabular}{|c|c|c|c|c|c|c|}
\hline \multirow{2}{*}{ Entry } & \multirow{2}{*}{ Aldehyde 2(a-k) } & \multirow{2}{*}{ Product $3(\mathrm{a}-\mathrm{k})$} & \multirow{2}{*}{$\begin{array}{l}\text { Time } \\
\text { (min) }\end{array}$} & \multirow{2}{*}{$\begin{array}{l}\text { Yield } \\
(\%)\end{array}$} & \multicolumn{2}{|c|}{ Melting points $\left({ }^{\circ} \mathrm{C}\right)$} \\
\hline & & & & & Obs. & References \\
\hline a. & & & 180 & 90 & $\begin{array}{c}275- \\
276\end{array}$ & $278[47]$ \\
\hline b. & & & 180 & 94 & $\begin{array}{c}190- \\
192\end{array}$ & $\begin{array}{c}194-196 \\
{[47]}\end{array}$ \\
\hline c. & & & 180 & 92 & $\begin{array}{c}262- \\
263\end{array}$ & $\begin{array}{c}263-264 \\
{[47]}\end{array}$ \\
\hline d. & & & 180 & 85 & $\begin{array}{c}172- \\
174\end{array}$ & $\begin{array}{c}174-176 \\
{[47]}\end{array}$ \\
\hline e. & & & 150 & 89 & $\begin{array}{c}220- \\
221\end{array}$ & $\begin{array}{c}217-219 \\
{[48]}\end{array}$ \\
\hline f. & & & 180 & 83 & 225 & $\begin{array}{c}225-227 \\
{[47]}\end{array}$ \\
\hline g. & & & 170 & 90 & 230 & $\begin{array}{c}230-232 \\
{[47]}\end{array}$ \\
\hline h. & & & 150 & 91 & $\begin{array}{c}257- \\
259\end{array}$ & $\begin{array}{c}260-261 \\
{[49]}\end{array}$ \\
\hline i. & & & 150 & 84 & 240 & $\begin{array}{c}237-238 \\
{[50]}\end{array}$ \\
\hline $\mathrm{j}$. & & & 150 & 92 & $\begin{array}{c}215- \\
216\end{array}$ & - \\
\hline $\mathrm{k}$. & & & 150 & 90 & $\begin{array}{c}220- \\
221\end{array}$ & - \\
\hline
\end{tabular}

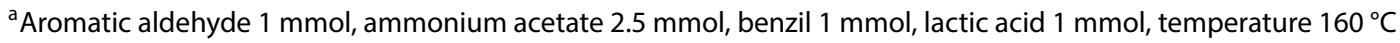




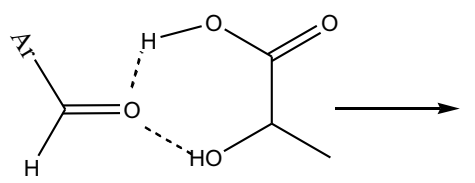<smiles>NC(=O)N[13CH]=C[18OH]</smiles>

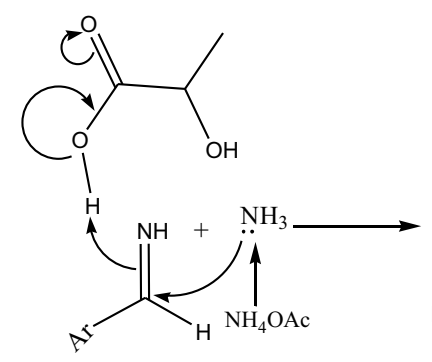<smiles>CC(O)C(=O)[O-]</smiles><smiles>NC(N)[AlH2]</smiles>

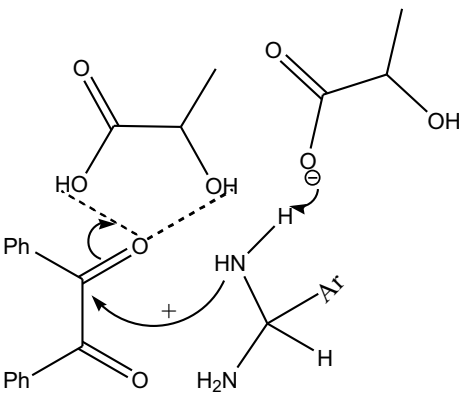

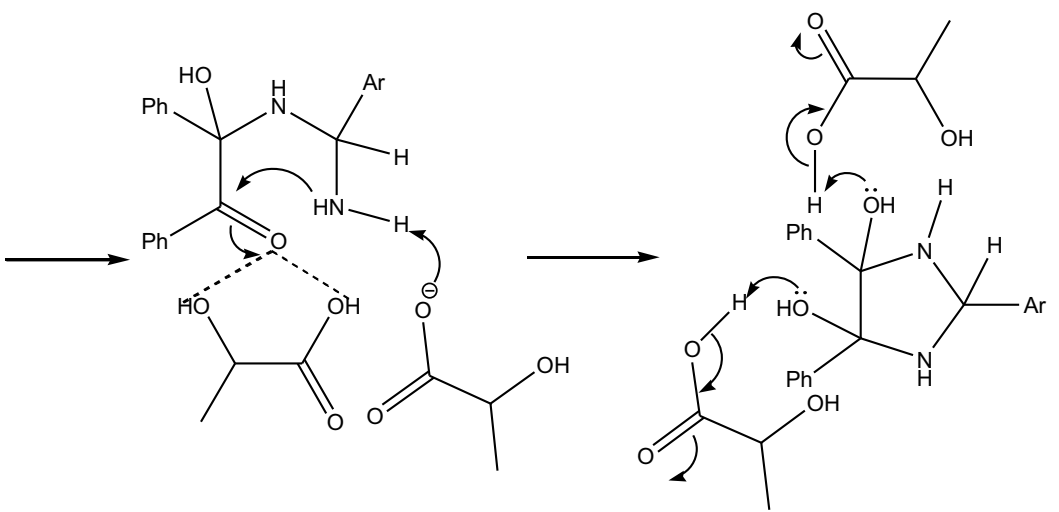

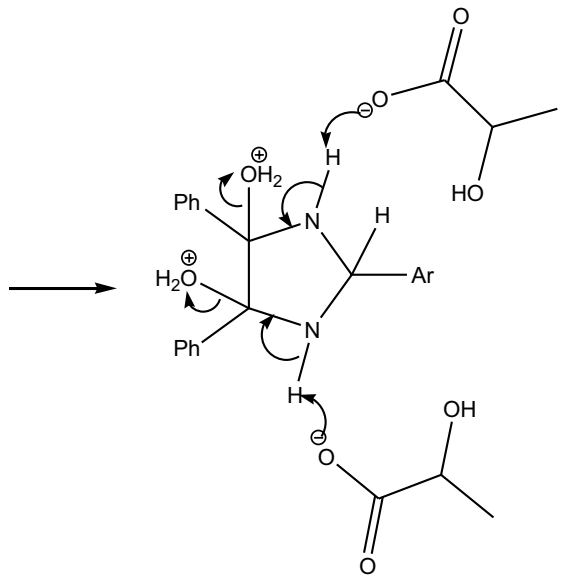

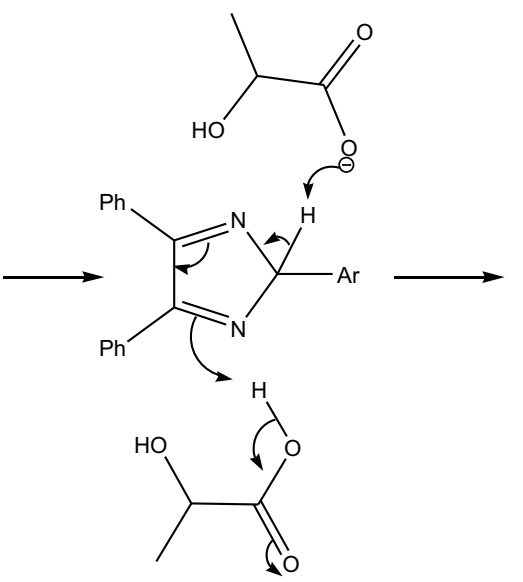<smiles>CC(O)C(=O)O</smiles><smiles>[Al]c1nc(-c2ccccc2)c(-c2ccccc2)[nH]1</smiles><smiles>CC(O)C(=O)[O-]</smiles>

Fig. 2 Plausible mechanism for the synthesis of 2,4,5-trisubstituted imidazoles using lactic acid

\section{Conclusion}

Herein we are reporting the efficient method using inexpensive, biodegradable and environmental benign green solvent for the synthesis of 2,4,5-trisubstituted imidazole. This method provides a better performance and higher product yield for aromatic aldehydes containing electron donating and electron withdrawing groups.
Acknowledgements Authors are thankful to the Principal, V. P. Mahavidyalaya, Vaijapur and the Principal, Deogiri College for providing laboratory facilities. Authors are also thankful to The Director, SAIF, Panjab University for providing spectral analysis.

\section{Compliance with ethical standards}

Conflict of interest The authors declare that they have no conflict of interest. 


\section{References}

1. Kumar V, Kaur K, Gupta GK, Sharma AK (2013) Pyrazole containing natural products: synthetic preview and biological significance. Eur J Med Chem 69:735-753. https://doi. org/10.1016/j.ejmech.2013.08.053

2. Kumar V, Mahajan MP (2011) In: Majumdar KC, Chattopadhyay SK (eds) Heterocycles in natural product synthesis. Pyrimidine and imidazole. Wiley-VCH, Weinheim, pp 507-533. https://doi. org/10.1002/9783527634880.ch14

3. Azizi SN, Shakeri P, Chaichi MJ, Bekhradnia A, Taghavi M, Ghaemy M (2014) The use of imidazolium ionic liquid/copper complex as novel and green catalyst for chemiluminescent detection of folic acid by Mn-doped ZnS nanocrystals. Spectrochim Acta Part A 122:482-488. https://doi.org/10.1016/j. saa.2013.11.036

4. Arshadi S, Bekhradnia AR, Ebrahimnejad A (2011) Feasibility study of hydrogen-bonded nucleic acid base pairs in gas and water phases-a theoretical study. Can J Chem 89:1403-1409. https://doi.org/10.1139/v11-124

5. Vessally E, Soleimani-Amiri S, Hosseinian A, Edjlalid L, Bekhradnia $A$ (2017) New protocols to access imidazoles and their ring fused analogues: synthesis from N-propargylamines. RSC Adv 7:7079-7091. https://doi.org/10.1039/c6ra25816f

6. Bastide M, Jouvert S, Bastid JM (1982) A comparison of the effects of several antifungal imidazole derivatives and polyenes on Candida albicans: an ultrastructural study by scanning electron microscopy. Can J Microbiol 28(10):1119-1126. https://doi. org $/ 10.1139 / \mathrm{m} 82-166$

7. Maier T, Schmierer R, Bauer K, Bieringer $H$, Buerstell $H$, Sachse B (1989) 1-substituted imidazole-5-carboxylic acid derivatives, their preparation and their use as biocides. U. S. Patent 4820 335, 1989, Chem. Abstr., 1989, 111, 19494w

8. Schmierer R, Mildenberger H, Buerstell H (1987) German Patent 361464, Chem. Abstr., (1988) 108: 37838

9. Mishra R, Ganguly S (2012) Imidazole as an anti-epileptic: an overview. Med Chem Res 21(12):3929-3939. https://doi. org/10.1007/s00044-012-9972-6

10. Robertson DW, Beedle EE, Lawson R, Leander JD (1987) Imidazole anticonvulsants: structure-activity relationships of [(biphenylyloxy)alkyl]imidazoles. J Med Chem 30(5):939-943. https:// doi.org/10.1021/jm00388a035

11. Puratchikodya A, Doble M (2007) Antinociceptive and antiinflammatory activities and QSAR studies on 2-substituted-4,5-diphenyl-1H-imidazoles. Bioorg Med Chem 15:1083-1090. https ://doi.org/10.1016/j.bmc.2006.10.025

12. Ali I, Lone MN, Aboul-Enein HY (2017) Imidazoles as potential anticancer agents. Med Chem Commun 8:1742-1773. https:// doi.org/10.1039/c7md00067g

13. Baroniya S, Anwer Z, Sharma PK, Dudhe R, Kumar N (2010) Recent advancement in imidazole as anti-cancer agents: a review. Der Pharm Sin 1(3):172-182

14. Abrahams SL, Hazen RJ, Batson AG, Phillips AP (1989) Trifenagrel: a chemically novel platelet aggregation inhibitor. $J$ Pharm Exp Therap 249(2):359-365

15. Cioc RC, Ruijter E, Orru RVA (2014) Multicomponent reactions: advanced tools for sustainable organic synthesis. Green Chem 16:2958-2975. https://doi.org/10.1039/c4gc00013g

16. Heravi MM, Bakhtiari K, Oskooie HA, Taheri S (2007) Synthesis of 2,4,5-triaryl-imidazoles catalyzed by $\mathrm{NiCl}_{2} \cdot 6 \mathrm{H}_{2} \mathrm{O}$ under heterogeneous system. J Mol Cat A 263:279-281. https://doi. org/10.1016/j.molcata.2006.08.070

17. Sangshetti JN, Kokare ND, Kotharkara SA (2008) Shinde DB (2008) Ceric ammonium nitrate catalysed three component one-pot efficient synthesis of 2,4,5-triaryl-1H-imidazoles.
J Chem Sci 120(5):463-467. https://doi.org/10.1007/s1203 9-008-0072-6

18. Sharma GVM, Jyothi Y, Lakshimi PS (2006) Efficient roomtemperature synthesis of Tri- and tetrasubstituted imidazoles catalyzed by $\mathrm{ZrCl}_{4}$. Synth Commun 36:2991-3000. https://doi. org/10.1080/00397910600773825

19. Karami B, Eskandari K, Farahi M, Barmas A (2012) An effective and new method for the synthesis of polysubstituted imidazoles by the use of $\mathrm{CrCl}_{3} \cdot 6 \mathrm{H}_{2} \mathrm{O}$ as a green and reusable catalyst: synthasis of some novel imidazole derivatives. J Chin Chem Soc 59:473-479. https://doi.org/10.1002/jccs.201100555

20. Patil VD, Sutar NR, Patil KP, Giddh P (2016) Synthesis of 2,4,5-triaryl-1 $1 \mathrm{H}$-imidazoles using anhydrous $\mathrm{Pb}(\mathrm{OAc})_{2}$ as a catalyst in $\mathrm{C}_{2} \mathrm{H}_{5} \mathrm{OH}$. Der Chem Sin 7(2):23-28

21. Safari J, Akbari Z, Naseh S (2016) Nanocrystalline $\mathrm{MgAl}_{2} \mathrm{O}_{4}$ as an efficient catalyst for one-pot synthesis of multisubstituted imidazoles under solvent-free conditions. J Saudi Chem Soc 20(1):S250-S255. https://doi.org/10.1016/j.jscs.2012.10.012

22. Nasr-Esfahani M, Montazerozohori M, Abdizadeh T (2015) Multicomponent synthesis of highly substituted imidazoles catalyzed by nanorod vanadatesulfuric acid. Chem Pap 69(11):1491-1499. https://doi.org/10.1515/chempap-2015-0156

23. Karami B, Eskandari K, Ghasemi A (2012) Facile and rapid synthesis of some novel polysubstituted imidazoles by employing magnetic $\mathrm{Fe}_{3} \mathrm{O}_{4}$ nanoparticles as a high efficient catalyst. Turk J Chem 36:601-614. https://doi.org/10.3906/kim-1112-49

24. Marzouk AA, Abbasov VM, Talybov AH, Mohamed SK (2013) Synthesis of 2,4,5-triphenyl imidazole derivatives using diethyl ammonium hydrogen phosphate as green, fast and reusable catalyst. World J Org Chem 1(1):6-10. https://doi.org/10.12691 /wjoc-1-1-2

25. Khosropour AR (2008) Synthesis of 2,4,5-trisubstituted imidazoles catalyzed by $[\mathrm{Hmim}] \mathrm{HSO}_{4}$ as a powerful Brönsted acidic ionic liquid. Can J Chem 86:254-269. https://doi.org/10.1139/ v08-009

26. Xia M, Lu Y-D (2007) A novel neutral ionic liquid-catalyzed solvent-free synthesis of 2,4,5-trisubstituted imidazoles under microwave irradiation. J Mol Catal A Chem 265(1-2):205-208. https://doi.org/10.1016/j.molcata.2006.10.004

27. Chary MV, Keerthysri NC, Vupallapati SVN, Lingaiah N, Kantevari $S$ (2008) Tetrabutylammonium bromide (TBAB) in isopropanol: an efficient, novel, neutral and recyclable catalytic system for the synthesis of 2,4,5-trisubstituted imidazoles. Catal Commun 9(10):2013-2017. https://doi.org/10.1016/j.catcom.2008.03.037

28. Shaabani A, Rahmati A, Aghaaliakbari B, Safaei-Ghomi J (2006) $1,1,3,3-\mathrm{N}, \mathrm{N}, \mathrm{N}^{\prime}, \mathrm{N}^{\prime}$-tetramethylguanidinium trifluoroacetate ionic liquid promoted efficient one-pot synthesis of trisubstituted imidazoles. Synth Commun 36:65-70. https://doi. org/10.1002/chin.200623121

29. Matsagar BM, Hossain SA, Islam T, Alamri HR, Alothman ZA, Yamauchi Y, Dhepe PL, Wu KC-W (2017) Direct production of furfural in one-pot fashion from raw biomass using Brønsted acidic ionic liquids. Sci Rep 7(1):1-7. https://doi.org/10.1038/ s41598-017-13946-4

30. Nemati F, Hosseini MM, Kiani H (2016) Glycerol as a green solvent for efficient, one-pot and catalyst free synthesis of 2,4,5-triaryl and 1,2,4,5-tetraaryl imidazole derivatives. J Saudi Chem Soc 20:S503-S508. https://doi.org/10.1016/j.jscs.2013.02.004

31. Chen Y, Wang R, Ba F, Hou J, Ding A, Zhou M, Guo H (2017) Synthesis of 2,4,5-triarylated imidazoles via three-component domino reaction under catalyst-free conditions. J Saudi Chem Soc 21(1):76-81. https://doi.org/10.1016/j.jscs.2016.03.001

32. Nalage SV, Kalyankar MB, Patil VS, Bhosale SV, Deshmukh SU, Pawar RP (2010) An efficient noncatalytic protocol for the synthesis of trisubstituted imidazole in polyethylene glycol using 
microwaves. Open Cat J 3:58-61. https://doi.org/10.2174/18762 14x01003010058

33. Li J-T, Chen B-H, Yan-Wei L, Xue-Li S (2012) Efficient Improved Synthesis of 2-Aryl-4,5-diphenylimidazole by Heating. Int J Adv Pharm Biol Chem 1(3):287-292

34. Shivani P, Sudhakar A, Gosh S (2013) One pot synthesis of tri and tetra substituted imidazole derivatives. Int J Pharm Biol Sci 3(4):270-277

35. Shelke K, Kakade G, Shingate B, Shingare M (2008) Microwaveinduced one-pot synthesis of 2,4,5-triarylimidazoles using glyoxylic acid as a catalyst under solvent-free conditions. Ras J Chem 1(3):489-494

36. Dhawale KD, Thorat NM, Patil LR (2017) An efficient and green synthesis of imidazoles using natural organic acids as promoter under solvent-free condition. Asian J Chem 29(8):1709-1712. https://doi.org/10.14233/ajchem.2017.20553

37. Häckl K, Kunz W (2018) Some aspects of green solvents. C R Chimie 21(6):572-580. https://doi.org/10.1016/j.crci.2018.03.010

38. Yang J, Tan J-N, Gu Y (2012) Lactic acid as an invaluable biobased solvent for organic reactions. Green Chem 14:3304-3317. https://doi.org/10.1039/c2gc36083g

39. Suresh Saini A, Kumar D, Sandhu JS (2009) Multicomponent ecofriendly synthesis of 3,4-dihydropyrimidine-2-(1H)-ones using an organocatalyst Lactic acid. Green Chem Lett Rev 2(1):29-33. https://doi.org/10.1080/17518250902973833

40. Kangani M, Hazeri N, Maghsoodlou MT (2017) Synthesis of pyrrole and furan derivatives in the presence of lactic acid as a catalyst. J Saudi Chem Soc 21(2):160-164. https://doi.org/10.1016/j. jscs.2015.03.002

41. Zhaleh S, Hazer N, Maghsoodlou MT (2016) Green protocol for synthesis of 2,3-dihydroquinazolin-4(1H)-ones: lactic acid as catalyst under solvent-free condition. Res Chem Intermed 42(7):6381-6390. https://doi.org/10.1007/s11164-016-2469-z

42. Sadeh FN, Hazeri N, Maghsoodlou MT, Lashkari M (2017) Efficient lactic acid-catalyzed route to naphthopyranopyrimidines under solvent-free conditions. Org Prep Proced Int 49:35-44. https://doi.org/10.1080/00304948.2017.1260395

43. Adrom B, Hazeri N, Maghsoodlou MT, Lashkari M, Fatahpour $M$ (2017) Green synthesis of 2-aryl-4-phenyl-quınazolıne derıvatives promoted by lactıc acıd. Maced J Chem Chem Eng 36(2):223-228. https://doi.org/10.20450/mjcce.2017.1137

44. Adrom B, Hazeri N, Maghsoodlou MT, Lashkari M, Fatahpour M (2017) Green synthesis of 2-aryl-4-phenyl-quınazolıne derivatives promoted by lactıc acıd. Maced J Chem Chem Eng 36(2):223-228. https://doi.org/10.20450/mjcce.2017.1137

45. Kangani M, Hazeri N, Yazdani-Elah-Abadi A, Maghsoodlou MT (2016) Lactic acid: an efficient and green catalyst for the onepot five-components synthesis of highly substituted piperidines. Polycycl Aromat Compd. https://doi.org/10.1080/10406 638.2016.1207686

46. Wagh RB, Nagarkar JM (2018) An efficient and sustainable protocol for oxidation of alcohols to carbonyl compounds. Tetrahedron Lett 59(37):3443-3447. https://doi.org/10.1016/j.tetle t.2018.08.011

47. Wang R, Liu C, Luo G (2010) A convenient synthesis of 2,4,5-triarylimidazoles catalyzed by $\mathrm{Y}(\mathrm{TFA})_{3}$. Green Chem Lett Rev 3(2):101-104. https://doi.org/10.1080/17518250903583680

48. Maleki B, Ashrafi SS (2014) N-Bromosuccinimide Catalyzed three component one-pot efficient synthesis of 2,4,5-triaryl-1H-imidazoles from aldehyde, ammonium acetate, and 1,2-diketone or a-hydroxyketone. J Mex Chem Soc 58(1):76-81. https://doi. org/10.29356/jmcs.v58i1.159

49. Safari J, Naseh S, Zarnegar Z, Akbari Z (2014) Applications of microwave technology to rapid synthesis of substituted imidazoles on silica-supported $\mathrm{SbCl}_{3}$ as an efficient heterogeneous catalyst. J Taibah Univ Sci 8:323-330. https://doi.org/10.1016/j. jtusci.2014.01.007

50. Nikoofar K, Dizgarani SM (2017) $\mathrm{HNO}_{3} @$ nano $\mathrm{SiO}_{2}$ : an efficient catalytic system for the synthesis of multi-substituted imidazoles under solvent-free conditions. J Saudi Chem Soc 21(7):787-794. https://doi.org/10.1016/j.jscs.2015.11.006

Publisher's Note Springer Nature remains neutral with regard to jurisdictional claims in published maps and institutional affiliations. 